\title{
Simulation of cold magnetized plasmas with the 3D electromagnetic software CST Microwave Studio ${ }^{\circledR}$
}

\author{
Fabrice Louche $^{1, \star}$, Alena Křivská ${ }^{1}$, André Messiaen $^{1}$, and Tom Wauters ${ }^{1}$ \\ ${ }^{1}$ Laboratory for Plasma Physics, ERM/KMS, EUROfusion Consortium Member, Brussels, Belgium, TEC Partner
}

\begin{abstract}
Detailed designs of ICRF antennas were made possible by the development of sophisticated commercial 3D codes like CST Microwave Studio ${ }^{\circledR}$ (MWS). This program allows for very detailed geometries of the radiating structures, but was only considering simple materials like equivalent isotropic dielectrics to simulate the reflection and the refraction of RF waves at the vacuum/plasma interface. The code was nevertheless used intensively, notably for computing the coupling properties of the ITER ICRF antenna. Until recently it was not possible to simulate gyrotropic medias like magnetized plasmas, but recent improvements have allowed programming any material described by a general dielectric or/and diamagnetic tensor. A Visual Basic macro was developed to exploit this feature and was tested for the specific case of a monochromatic plane wave propagating longitudinally with respect to the magnetic field direction. For specific cases the exact solution can be expressed in 1D as the sum of two circularly polarized waves connected by a reflection coefficient that can be analytically computed. Solutions for stratified media can also be derived. This allows for a direct comparison with MWS results. The agreement is excellent but accurate simulations for realistic geometries require large memory resources that could significantly restrict the possibility of simulating cold plasmas to small-scale machines.
\end{abstract}

\section{Introduction}

During the two last decades a significant number of Ion Cyclotron Resonance Frequency (ICRF) systems for heating, current drive and wall conditioning have been developed. The accurate design and the geometry optimisation of these systems were made possible with the development of commercial softwares like CST Microwave Studio ${ }^{\circledR}[1]$ (MWS), or codes developed in the community like TOPICA[2] and ANTITER II[3]. MWS is based on the "Finite Integration Technique" (FIT) and uses special broadband frequency sweep techniques in order to derive the full spectrum from a limited number of frequency samples. Solids are meshed with a tetrahedral meshing algorithm with iterative refinement techniques. One of the main advantages of MWS is the user-friendly graphical interface that allows to develop very accurate 3D geometries. It has been used for the design and the optimisation of the ICRF antennas for devices like JET [4], ITER[5], W7-X[6], TOMAS[7] and IShTAR[8] notably. The input impedance matrices computed by the program were used to develop matching systems, while the RF fields and currents maps were integrated to evaluate thermal properties and plasma production possibilities. Unfortunately all these evaluations did not incorporate plasma loading. Nevertheless it was proven numerically and experimentally [9] that a dielectric load can fairly simulate plasma loading, with a proper choice of dielectric permittivity for

\footnotetext{
^e-mail: fabrice.louche@rma.ac.be
}

a given set of plasma parameters $\left(K_{D} \sim c^{2} / v_{A}{ }^{2}\right.$, where $c$ is the light velocity and $v_{A}$ is the Alfven velocity).

General tensor materials can now be incorporated into MWS and we have written a Visual Basic macrocommand that allows to create a cold magnetized plasma. As long as thermal effects can be neglected, i.e. far from resonances and confluence regions, the propagation of waves in plasmas can be described by the cold plasma model. In this model we consider a plasma immersed in a static magnetic induction field $\overrightarrow{B_{0}}$, aligned with the $z$-axis of the Cartesian coordinate system. The components of the cold-plasma dielectric tensor $\boldsymbol{\varepsilon}$ were given by Stix [10]. In the ICRF, when $\omega \sim \omega_{c i}$ and $\omega_{c e} \gg \omega_{c i}$, the perpendicular term can be approximated by $\varepsilon_{\perp} \approx-\sum_{i} \omega_{p i}^{2} /\left(\omega^{2}-\omega_{c i}^{2}\right)$ where the sum is limited to the ion contributions, the offdiagonal term becomes $\varepsilon_{x y} \approx \sum_{s} \omega_{p s}^{2} \Omega_{c s} /\left(\left(\Omega_{c s}^{2}-\omega^{2}\right) \omega\right)$, while the parallel term includes only the electron contribution.

\section{Definition of a test case}

The Stix's tensor for a cold plasma was implemented into MWS via a macro-command with an intuitive user interface. The macro must first be tested in cases for which an exact or analytical solution can be derived. The routine must reproduce the classical features of cold plasma waves, like the co-existence of two modes (bi-refringence) with wavelengths given by the solutions of the dispersion equation. But we also want to compare the field maps computed by MWS with analytical solutions. 
We therefore consider a linearly polarized monochromatic plane wave $E_{y}$ excited in vacuum and incident to a given plasma medium. The plasma is bounded by a metallic wall or by an open boundary that imposes a zero reflection condition (see figure 1). For this latter case a perfectly matched layer (PML) must be defined (see Sect. 3.2). The wave propagation is purely longitudinal with respect to the static magnetic field $\overrightarrow{B_{0}}=B_{0} \overrightarrow{1}_{z}$. Analytical solutions can be obtained for homogeneous plasmas, stratified plasmas, and for various types of continuous plasma profiles. This allows to directly compare MWS results with analytical solutions and to identify discrepancies coming from the formulation of the numerical problem within MWS.

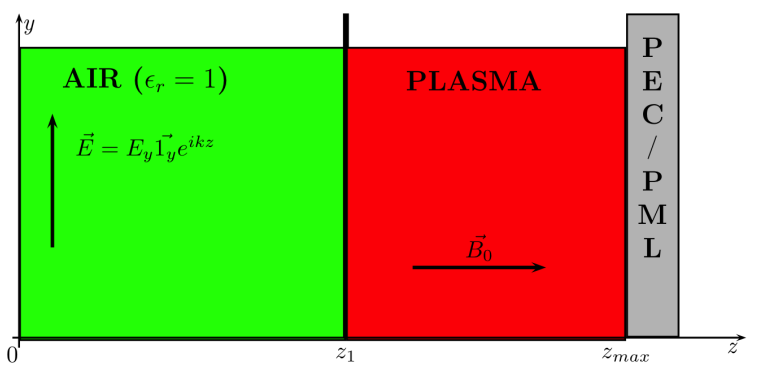

Figure 1. 1D model of a linearly polarized monochromatic plane wave $E_{y}$ with longitudinal propagation w.r.t. the magnetic field direction

When the propagation is parallel to the magnetic field, the two solutions to the dispersion equation reduce to $k_{ \pm}=k_{0} \sqrt{\epsilon_{\perp} \pm \epsilon_{x y}}=k_{0} \sqrt{\epsilon_{ \pm}}$, with $k_{0}=\omega / c$ and the components of the dielectric tensor being given in Sec. 1. Both modes are characterized by the absence of electric and magnetic fields component in the direction of propagation $\left(E_{z}, H_{z}=0\right)$. They are circularly polarized: $\left(E_{x} / E_{y}\right)_{ \pm}=\mp i$ for respectively Left Handed Circular Polarization (LHCP) and Right Handed Circular Polarization (RHCP), the latter being mostly evanescent for typical tokamak plasma parameters in the ICRF. The problem of the longitudinal propagation of a plasma wave in a magnetized plasma can be reduced to the propagation of two circularly polarized plane waves in isotropic dielectrics with permittivities $\epsilon_{+}$and $\epsilon_{-}$respectively. The methodology for the classical 1D transmission/reflection problem of a plane wave incident to layered or continuous dielectric media can be exploited for treating the case of a longitudinal plasma wave with an appropriate change of frame from Cartesian coordinates to circular coordinates.

All the results presented in the following sections will be for a Deuterium-Tritium plasma with equal ion concentrations. Two sets of parameters will be considered: "plasma\#1" with $\mathrm{B}_{0}=2.5 \mathrm{~T}$ and $\mathrm{n}_{\mathrm{e}}=510^{13} \mathrm{~cm}^{-3}$ and "plasma\#2" with $\mathrm{B}_{0}=5 \mathrm{~T}$ and $\mathrm{n}_{\mathrm{e}}=910^{13} \mathrm{~cm}^{-3}$. In these cases the RHCP mode is mostly evanescent, it only becomes propagative at low frequency for the high density case "plasma\#2".

\section{MWS results for 1D longitudinal propagation}

We define the $z$-axis as the direction of propagation and inhomogeneity (see figure 1 ). The linearly polarized $E_{y}$ is excited at $z=0$. The boundary at $z=z_{\max }$ will be discussed in the following sections. Periodic boundary conditions are imposed at the 4 other boundaries: this guarantees that the numerical problem is strictly equivalent to a 1D propagation case. An important parameter for MWS models is the mesh density. The accuracy of the solution of the numerical problem as stated in MWS is strongly dependent on the meshing of the spatial domain. A fine mesh is needed to accurately describe the spatial variations of the electromagnetic fields. Each medium (plasma, air, ...) will be defined by its specific sub-grid tuned by a parameter $\delta_{z}$ defined as the number of cells needed to discretize a characteristic reference wavelength in the medium: the side of a cell will roughly be $\lambda_{r e f} / \delta_{z}$.

\subsection{Subcase \#1: Homogeneous plasma bounded by a metallic wall}

We begin with the case of a $1 \mathrm{D}$ plasma with length $l_{2}$ bounded by a perfect metallic boundary at $z=z_{\max }$. In each medium (air, plasma) the solution is a superposition of a forward and a backward wave. At the air/plasma interface the reflection coefficient is given by $\Gamma_{1}=\left(Z_{p l, \pm}-\eta_{0}\right) /\left(Z_{p l, \pm}+\eta_{0}\right)$, with $\eta_{0}$ is the intrinsic impedance of vacuum, and $Z_{p l, \pm}=-i \eta_{ \pm} \tan k_{ \pm} l_{2}$ is the impedance viewed towards the plasma [11].

For the specific problem of a plane wave reflected at a metallic wall, two successive voltage nodes are separated by a distance equal to $\lambda_{\text {ref }}=\lambda / 2$ in each medium. This is the minimum length that must be accurately resolved by the parameter $\delta_{z}$. Figure 2 displays the amplitude of some components of the electric field as a function of $\delta_{z}$. We see that an excellent agreement between the MWS results and the analytical solution is obtained, but it requires a very fine mesh defined by $\delta_{z}>300$ to reduce discrepancies. For the set of parameters discussed here this leads to a sub-millimetric size of a typical elementary mesh. Also it should be noted that for the cases where both modes are propagative the bi-refringence is correctly described by MWS (see figure 2-(c)).

\subsection{Subcase \#2: Infinite homogeneous plasma}

In usual antenna modeling with MWS and dielectric loading the single pass absorption in a plasma bulk was simulated by a purely outgoing wave condition at boundaries of the simulation domain. In our 1D test case this is an elementary problem to solve analytically, but it cannot be straightforwardly implemented into MWS for plasmas. Indeed the so-called "open boundary conditions" emulating radiating boundaries in MWS cannot be used in contact with anisotropic materials. The idea of a "Perfectly Matched Layer" (PML) was proposed in [12] and extended by many authors in specific situations. In particular, the use of PMLs for magnetized plasmas has been studied in 

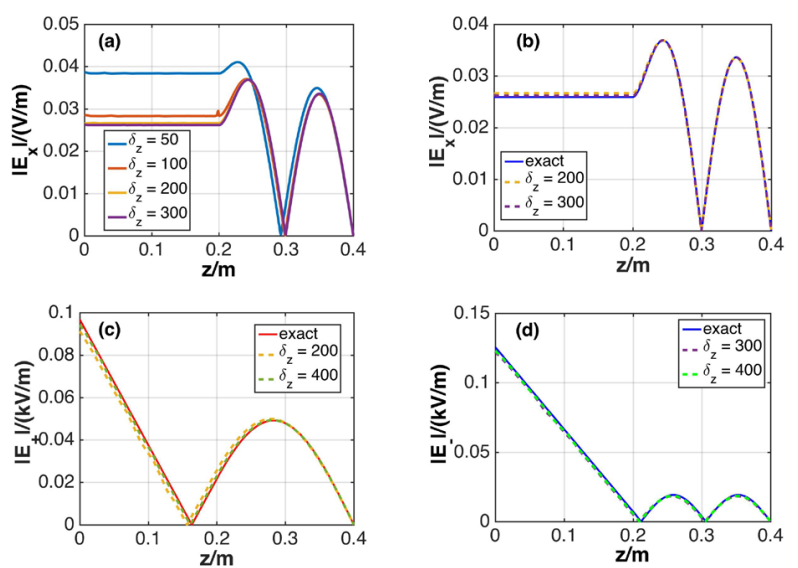

Figure 2. Amplitude of the electric field for various levels of mesh accuracy: (a) $E_{X}$ component at $50 \mathrm{MHz}$ for plasma\#1 at low mesh density; (b) $E_{X}$ component at $50 \mathrm{MHz}$ for plasma\#1 at high mesh density; (c) $E_{+}$at $20 \mathrm{MHz}$ component for plasma\#2; (d) $E_{-}$component at $20 \mathrm{MHz}$ for plasma\#2.

[13] and for ICRF plasmas in [14]. A PML is an additional boundary layer put at the edge of the computation domain where perfect absorption is desired. This layer is made of an artificial lossy material whose properties are tailored in such a way to absorb and exponentially attenuate any incoming wave to guarantee zero-reflection at the boundary with the plasma medium, while the PML is followed by a perfect metallic boundary.

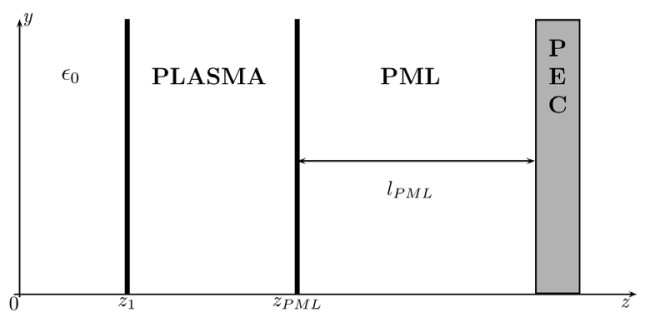

Figure 3. Definition of 1D PML

For our simplified 1D case the PML is characterized by a transformed dielectric tensor given by

$$
\boldsymbol{\varepsilon}_{\boldsymbol{P M L}}=\left(\begin{array}{ccc}
\varepsilon_{\perp} S_{z} & -i \varepsilon_{x y} S_{z} & 0 \\
i \varepsilon_{x y} S_{z} & \varepsilon_{\perp} S_{z} & 0 \\
0 & 0 & \varepsilon_{\|} / S_{z}
\end{array}\right)
$$

and a similar formula for the (relative) diamagnetic tensor. A common choice for the stretching function is to use a polynomial form as:

$$
S_{z}(z)=1+\left(S_{z}^{\prime}+i S_{z}^{\prime \prime}\right)\left(\frac{z-z_{P M L}}{l_{P M L}}\right)^{p_{z}}
$$

where $z_{P M L}, l_{P M L}, p_{z}, S^{\prime}$ and $S^{\prime \prime}$ are respectively the location of the plasma/PML interface, the PML depth (see fig. 3), the order of the stretching function and the stretch parameter (real and imaginary part). The absorption of the LHCP wave in the PML will be optimized if $p_{z}=1$ and the imaginary part of the stretching is sufficiently large, and for sufficiently long PML to allow damping.

Before we implement the PML medium into CST MWS, we must discuss an additional approximation. The PML is a medium where the dielectric properties vary continuously in space. As such media cannot be modelled in MWS, the continuous PML will be approximated by a stratified medium with each layer having the same thickness and being characterized by constant PML electric and magnetic permittivities. The Helmholtz equation for a longitudinal plasma wave in a stratified medium can be solved by an iterative method which involves to compute recursively the reflection coefficients at each interface [11]. We can then obtain the exact reflection coefficient at the plasma/PML boundary for each mode and compare to the one of a perfect PML. This gives a precise way to assess the minimum number of layers needed to satisfactorily discretize the PML. Typical results can be seen in figure 4-(a): we note that a large number of layers is obviously needed for reproducing the variations and amplitude of the field magnitude. If this number is low an artificial high amplitude standing wave pattern is visible. The important parameter here is the local wavelength in the PML given by $\lambda_{P M L}(z)=2 \pi k_{P M L}=\lambda_{ \pm} / S_{z}(z)$. If we plot the reflection coefficient as a function of the number of layers per wavelength, where the reference value is the minimum $\lambda_{P M L}$ at the back of the PML, we observe satisfactory convergence for all stretching functions when the medium is approximated by at least 100 layers (less that $5 \%$ error): figure 4-(c). In practical situations where MWS is used the choice of the number of layers will be a compromise between accuracy of the absorption by the PML and reasonable thickness for the layers. Indeed for a case with very good absorption like $S_{z}=1+3 i$ and $p_{z}=1$, the minimum wavelength is $3 \mathrm{~cm}$ only which would lead to layers of sub-millimeter thickness. If we look at the value of the reflection coefficient for 10 layers we obtain a reflection of $310^{-6}$, which is sufficient to guarantee an effective PML. This is a pragmatic choice which will make the MWS models much more reasonable.
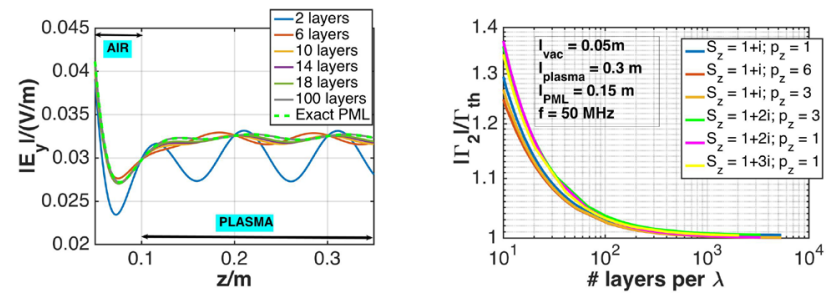

Figure 4. Study of stratified PML; (a) electric field profile for $S_{z}=1+i, p_{z}=1$ and $l_{P M L}=0.15 \mathrm{~m}$,compared with the solution for the continuous PML for various number of layers in the PML; (b) ratio between the reflection coefficient at the plasma/PML interface for stratified PML and continuous PML .

Let us now come to the MWS model. Due to the reduction of the wavelength along the PML we have incorporated a specific sub-meshing for this medium, with an increased meshing density. While we use the same $\delta_{z}=300$ as defined previously to tune the plasma mesh- 
ing (for $\lambda_{\text {ref }}=\lambda \simeq 0.2 \mathrm{~m}$ ), we now use for the PML a reduced reference wavelength. Varying this quantity gives results such as figure 5. We obtain an excellent agreement with the exact solution for a stratified PML. But clearly this comes at the price of a substantially finer mesh than in the plasma. It should also be noted that an almost perfect agreement also requires a very detailed mesh in the vacuum/air region. Reducing the length of the elementary vacuum mesh $z_{v a c}$ from $10 \mathrm{~mm}$ to $5 \mathrm{~mm}$ was needed to avoid spurious oscillations in this region.
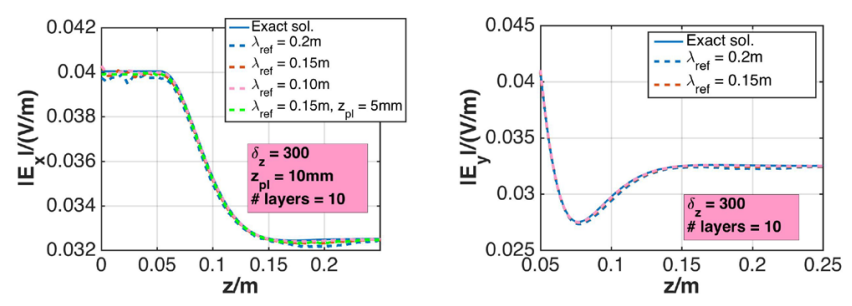

Figure 5. Electric field components $E_{x}$ and $E_{y}$ computed by MWS for a given PML $\left(S_{z}=1+3 i, p_{z}=1\right.$ and $\left.l_{P M L}=150 \mathrm{~mm}\right)$ for various reference wavelengths for the meshing in the PML. "Plasma \#1" set of parameters. Frequency is $50 \mathrm{MHz}$.

\subsection{Subcase \#3: Linear density profile}

We finally consider the case of a linearly increasing density profile followed by a plasma bulk at constant density: see figure 6. For this case an exact analytical solution for the fields in each medium can be derived. The field in the linearly varying density region is given by the sum of two Airy functions [15]: $E_{2}(z)=C_{1} A i(\xi)+C_{2} B i(\xi)$ with $\xi=-\left(k_{0}^{2} \alpha\right)^{1 / 3}\left(z+\alpha^{-1}\right)$ and $\alpha$ is the slope of the density profile. The two constants $C_{1}$ and $C_{2}$, the reflection coefficient in the air region $\Gamma_{1}$, and the transmitted amplitude $E_{0}^{\prime}$ can be exactly computed by imposing the continuity of both the electric and the magnetic field at each interface.

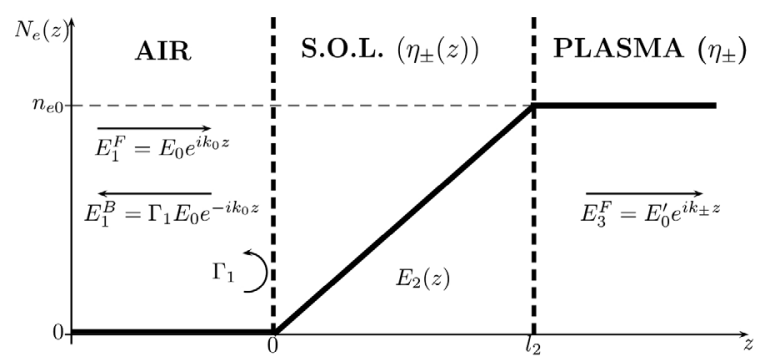

Figure 6. Definition of the parameters for linear density profile bounded by a homogeneous plasma.

We have run MWS simulations for various types of profiles, characterized by different slopes, at $50 \mathrm{MHz}$. The mesh parameter is $\delta_{z}=300$, while the usual PML stretching parameter $1+3 i$ is used, with 10 layers in the PML. We compare the solution for an increasing number of layers in the profile region with the exact analytical solution in figure 7. A minimum number of 50 layers is needed to obtain the best agreement. It should be noted that the profile discussed here does not lead to any resonance or cut-off at
$50 \mathrm{MHz}$ and therefore a homogeneous stratification of the profile is possible. In a situation where the fast wave encounters a cut-off, an adaptive algorithm of stratification will be necessary. This is still under study.
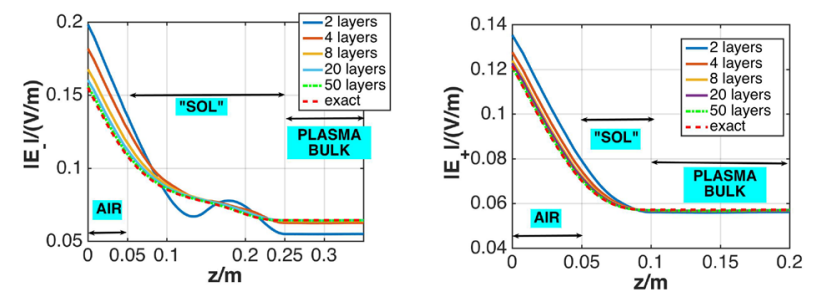

Figure 7. LHCP component of the electric field for various number of layers in the density profile region, compared with the analytical solution. Frequency is $50 \mathrm{MHz}$. Left: plasma \#1; right: plasma\#2 (higher density).

\section{Conclusions}

The work presented here is a first step in the direction of more realism in the predictions obtained from MWS. The plasma module developed for this study has been benchmarked for a 1D model of plasma and the results obtained were in perfect agreement with the exact analytical solutions. Nevertheless the accuracy of the spatial discretization of the plasma material needed to reach this agreement is extremely high and the modeling of realistic 3D antennas facing magnetized plasmas should require huge hardware resources, in particular for cases with radiative boundaries where a PML must be added to the simulation. The possibility to use a parallelised version of MWS will be exploited in a near future.

\section{References}

[1] CST STUDIO SUITE ${ }^{\circledR}$, CST AG, Germany

[2] V. Lancellotti et al., Nucl. Fusion 46, S476 (2006)

[3] A. Messiaen et al., Nucl. Fusion 50, 025026 (2010)

[4] P. Lamalle et al., Nucl. Fusion 46, 432 (2006)

[5] F. Louche et al., Nucl. Fusion 51, 103002 (2011)

[6] F. Louche et al., Fusion Eng. Des. 96-97, 508 (2015)

[7] F. Louche et al., Fusion Eng. Des. (2017) http://dx.doi.org/10.1016/j.fusengdes.2017.04.123.

[8] F. Louche et al. AIP Conf. Proc., 1689 070016, 2015.

[9] A. Messiaen et al. Fusion Eng. Des., 86 978, 2011.

[10] T.H. Stix, Waves In Plasmas (New-York: AIP, 1992)

[11] S. Orfanidis, Electromagnetic Waves and Antennas (ECE Department Rutgers University, www.ece.rutgers.edu/orfanidi/ewa/, 2014)

[12] J. Berenger, J. Comput. Phys. 114, 185 (1994)

[13] N. Gondarenko, J. Comput. Phys. 194, 481 (2004)

[14] J. Jacquot et al., Plasma Phys. Control. Fusion 55, 115004 (2013)

[15] K. Yeh, C.H. Liu, Theory of Ionospheric Waves (Academic Press, 1972) 